\title{
Scientia Agropecuaria
}

\section{Tipología de productores y sostenibilidad de cultivos en Alto Urubamba, La Convención - Cusco}

\section{Farmers typology and crops sustainability in Alto Urubamba, La Convencion - Cusco}

\author{
Isaías Merma $^{1}$; Alberto Julca ${ }^{2}$ \\ 1 Universidad Nacional San Antonio Abad del Cusco - Perú. Facultad de Ciencias Agrarias Tropicales de Quillabamba, \\ La Convención (i_merma@hotmail.com). \\ 2 Universidad Nacional Agraria La Molina. Facultad de Agronomía. Departamento Académico de Fitotecnia \\ (ajo@lamolina.edu.pe).
}

Recibido 11 febrero 2012; aceptado 03 junio 2012

\begin{abstract}
Resumen
La investigación se llevó a cabo en la región geográfica del Alto Urubamba, provincia de La Convención, Cusco - Perú. El objetivo fue identificar tipos de productores y evaluar la sostenibilidad de cultivos en fincas agrícolas de selva alta. Para la tipología se aplicó encuestas a una muestra de 106 productores tanto en términos biofísicos como socio-económicos analizándose la información mediante la estadística descriptiva. Luego se identificó los tipos de productores mediante el análisis multivariado utilizando variables previamente seleccionadas. Asimismo se evaluó la sostenibilidad de ocho cultivos tropicales; para esto, se seleccionó tres fincas por cultivo de un total de 24 fincas evaluadas. Se utilizó índices prácticos de calidad del suelo y salud del cultivo con una valoración de 0 a 10 y se calificó con la participación de los productores. Los resultados muestran que en la zona existen tres tipos de productores según su eficiencia en el manejo de recursos y su lógica económica. Los cultivos de té (6.65) y mango (6.50) obtienen los valores más altos de sostenibilidad, seguidos del café (6.25), cacao (6.25), cítricos (5.50), plátano (5.45) y coca (5.10). La papaya (4.60) muestra un valor menor a cinco, lo que califica a este cultivo como no sustentable para las condiciones en estudio.
\end{abstract}

Palabras clave: Tipología de productores, sostenibilidad de cultivos, selva alta, calidad del suelo, salud del cultivo.

\begin{abstract}
The research was conducted in the geographical region of Alto Urubamba, province of La Convencion, Cusco - Peru. The objective was to identify types of farmers and evaluate crops sustainability on farms of high forest. Surveys were applied to a sample of 106 farmers in both biophysical and socio-economic terms in order to identify typology; this information was analyzed through descriptive statistics. Multivariate analysis using preselected variables was performed to identify types of farmers. In addition, sustainability of eight tropical crops was evaluated; for this purpose, three farms for each crop were selected from 24 evaluated farms. Practical indicators of soil quality and crop health with a valuation from 0 to 10 were used; farmers participated during this evaluation. The results show that there are three types of farmers according to their efficiency in resources management and their economic logic. The crops of tea (6.65) and mango (6.50) obtained the highest values of sustainability, followed by coffee (6.25), cocoa (6.25), citrus (5.50), banana (5.45) and coca (5.10). Papaya (4.60) shows a value less than five; therefore, is considered as unsustainable according to local conditions.
\end{abstract}

Keywords: Farmers typology, crops sustainability, high forest, soil quality, crop health.

\section{Introdución}

Los estudios sobre tipología de productores y evaluación de la sostenibilidad de cultivos tropicales en

\footnotetext{
* Autor para correspondencia

Email: i_merma@hotmail.com (I. Merma)
}

selva alta en suelos con limitaciones de ladera y sequía estacional son escasos, encontrándose pocas referencias en la literatura científica. 
El análisis de los sistemas agrarios con base en la sustentabilidad se centra especialmente a nivel de finca (Lovell et al., 2002; Pacini et al., 2003; LópezRidaura et al., 2005), porque en este ámbito es donde se expresan más nítidamente las limitantes a la producción agrícola, lo que permite abordar y discutir acerca de las barreras técnicas, ecológicas y sociales más relevantes (Guzmán y Alonso, 2007).

La tipología consiste en identificar grupos de productores con características similares (Hart, 1990; Malagón y Prager, 2001). La tipificación permite agrupar a la diversidad de productores, según su lógica económica, que es expresión del tipo de recursos que posee, de su habilidad y experiencia tecnológica y de las limitantes y potencialidades de la zona (FAO / USAC, 1995).

La tipología es el resultado de un análisis y de un razonamiento previo en base a observaciones de campo y entrevistas con los agricultores. El objetivo es formar grupos de productores, sobre la base de criterios cualitativos de homogeneidad que tengan sentido, en el cual se establezcan diferencias claras entre unos y otros, dejando de lado las heterogeneidades secundarias (Apollin y Eberhart, 1999)

La elaboración de tipologías parte de un interés operativo: busca "simplificar la diversidad", al identificar grupos (tipos) de sistemas de producción que presenten potencialidades y restricciones similares frente a uno $o$ varios elementos seleccionados (Amador et al., 1995).

La sostenibilidad se define como la habilidad de un sistema de mantener la productividad aun cuando sea sometido a "stresess o perturbaciones" (Conway, 1994). Según Dixon y Fallon (1989), sustentabilidad (o más correctamente, cosecha sostenida) significa utilizar el recurso sin reducir su stock físico. Para Masera et al. (1999) la sustentabilidad puede definirse como el mantenimiento de una serie de objetivos (o propiedades) deseados a lo largo del tiempo.

Cuando la evaluación de sostenibilidad se aplica en varios cultivos resulta muy útil para los agricultores porque les permite comprender las razones por las cuales algunos cultivos tienen una respuesta superior a otros y que medidas implementar para mejorar aquellos aspectos en que los indicadores mostraron valores bajos (Altieri y Nicholls, 2002).

El desempeño de las fincas y la capacidad de gestión de los agricultores dependen de los cultivos/crianzas y la tecnología empleada, por ello es importante evaluar los cultivos prevalentes que maneja el agricultor para conocer su grado de sostenibilidad.

La evaluación de la sostenibilidad de sistemas complejos, como los agrarios, requiere la aplicación de nuevos enfoques analíticos e indicadores (Girardin et al. 1999; Pretty, 2008). El objetivo final de los investigadores que desarrollan y promueven técnicas de manejo sostenible, es llegar a diseñar agroecosistemas con gran resistencia a plagas, buena capacidad de reciclaje y de retención de nutrimentos, así como altos niveles de biodiversidad (Gliessman, 1998).

Un desafío que enfrentan tanto agricultores, extensionistas e investigadores es saber ¿cuando un agroecosistema puede ser considerado saludable?, o ¿en qué estado de salud se encuentra? Los investigadores que trabajan en agricultura sostenible han propuesto una serie de indicadores de sostenibilidad para evaluar el estado de los agroecosistemas con cultivos (Gómez et al., 1996; Masera et al., 1999). Algunos indicadores consisten en observaciones o mediciones que se realizan a nivel de finca para determinar la fertilidad y conservación del suelo y si las plantas están sanas, vigorosas y productivas.

Un sistema más diversificado, con un suelo rico en materia orgánica y biológicamente 
activo es considerado un sistema no degradado, robusto y productivo. En otras palabras, un agroecosistema con un cultivo prevalente rico en biodiversidad, la cual a partir de una serie de sinergismos contribuye a la fertilidad edáfica, la fitoprotección y la productividad del sistema, se considera sustentable o saludable (Fernández y Muschler, 1999).

La medición de la sostenibilidad de cultivos es una tarea necesaria. Existe una metodología rápida para evaluar la calidad del suelo y la salud del cultivo en café, usando indicadores sencillos y prácticos de utilizar por los agricultores. Además son precisos y fáciles de interpretar, sensitivos a los cambios ambientales y al impacto de las prácticas de manejo; integran propiedades físicas, químicas y biológicas del suelo y pueden relacionarse con procesos del ecosistema (Altieri y Nicholls, 2002).

El objetivo del presente estudio fue identificar tipos de productores y evaluar la sostenibilidad de cultivos en fincas agrícolas de selva alta en la región del Alto Urubamba, La Convención - Cusco.

\section{Materiales y métodos}

\subsection{Lugar de estudio}

El estudio se llevó a cabo en el Alto Urubamba, La Convención - Cusco; zona de selva alta, en la cual hay agricultores que se distinguen por la producción de cultivos para la venta como café, cacao, té, coca, frutales tropicales y productos alimenticios para el consumo.

El Alto Urubamba es una región geográfica de la provincia La ConvenciónCusco. Localizada en la parte sur oriental del Perú, entre los paralelos $11^{\circ} 15^{\prime}$ y $13^{\circ}$ $30^{\prime}$ Latitud Sur y los meridianos $72^{\circ}$ y $74^{\circ}$ Longitud Oeste. La extensión territorial es de $6525 \mathrm{Km}^{2}$, comprende la cuenca alta del río Urubamba que recorre la provincia de sur a norte hacia la vertiente atlántica, formando parte del sistema hidrográfico del río Amazonas.
La región se encuentra en un rango altitudinal entre 600 a $2200 \mathrm{msnm}$; con una temperatura media anual de $25.4{ }^{\circ} \mathrm{C}$; la precipitación anual está entre 989 y 1200 $\mathrm{mm}$, con una época lluviosa de octubre a marzo y una época seca de abril a septiembre que diferencia seis meses secos con menos de $100 \mathrm{~mm} / \mathrm{mes}$. La fisiografía dominante (selva alta) puede definirse como vertiente escarpada, ondulada y accidentada con gradientes y laderas entre 15 y $70 \%$.

La metodología del estudio comprende: (a) recoger información de los predios de acuerdo a las dimensiones de análisis agroecológica, técnico-productiva y socioeconómica (b) sistematizar y analizar la información (c) identificar los tipos de productores; (d) evaluar las propiedades de calidad del suelo y salud del cultivo en los agroecosistemas (e) determinar la sustentabilidad de los cultivos prevalentes.

\subsection{Tipología}

La población muestral fue de 106 productores, a los cuales se aplicaron encuestas en base a cuestionarios previamente elaborados. La información se ordenó y se analizó en sus tres dimensiones: biofísico, económico y social. El análisis comprende la elaboración de una base de datos y la aplicación del programa SPSS V.15 con la finalidad de caracterizar a los productores encuestados.

Para identificar tipos de productores se efectuó el "análisis multivariado" utilizando el programa MINITAB V.14. Para esto, se seleccionó variables como: escolaridad del productor, actividad económica principal, propiedad y extensión de la finca, ingreso económico anual, acceso a crédito y riego, cultivos prevalentes, sistemas de producción y factores que influyen en la actividad productiva de la finca.

Como resultado se obtiene la agrupación de productores en tipos diferenciados o cluster. 


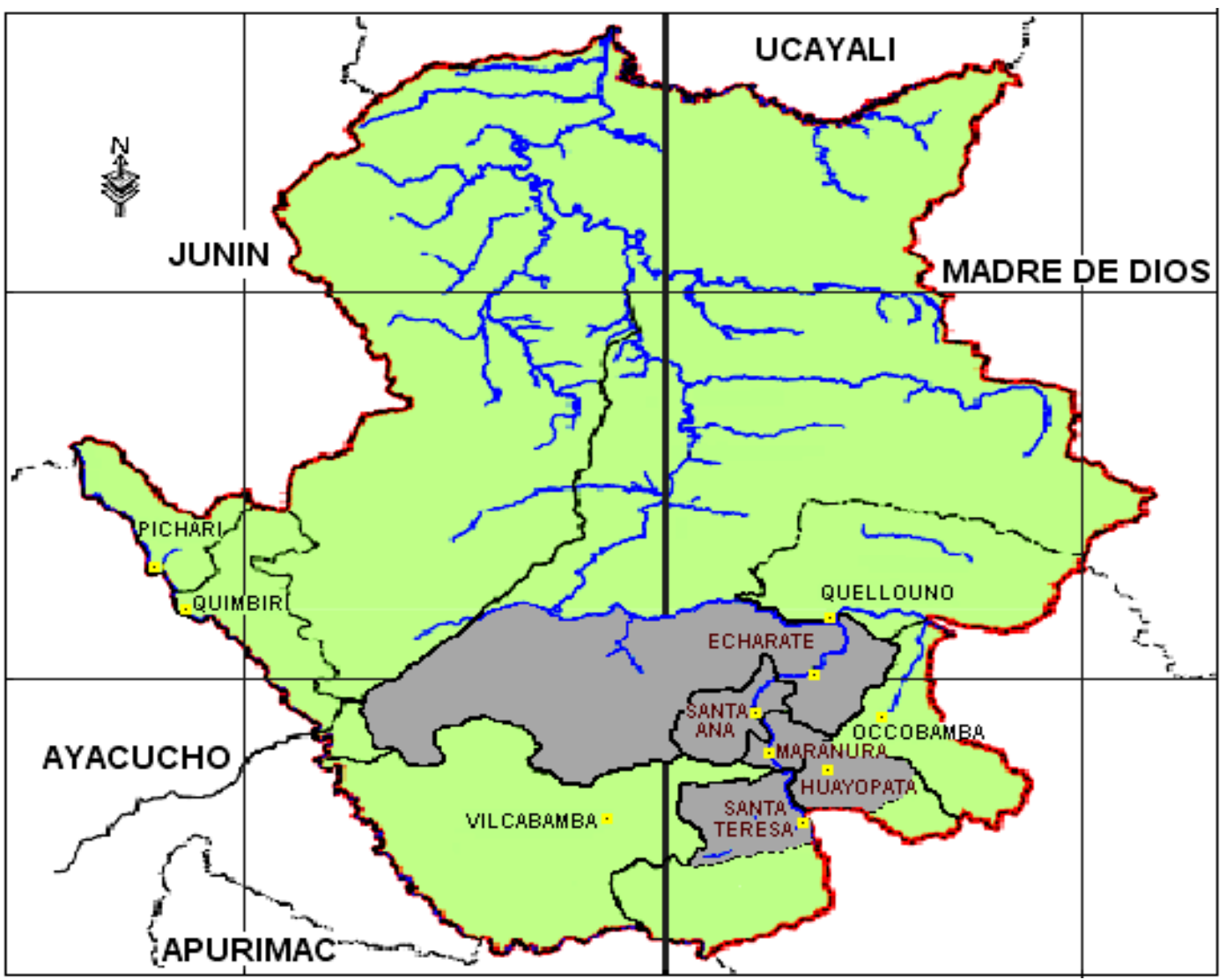

Figura 1. Mapa de la provincia La Convención (verde) y la región del Alto Urubamba (de color gris).

\subsection{Evaluación de la sustentabilidad}

Para evaluar la sustentabilidad de cultivos, se utilizó “el método agroecológico rápido para la evaluación de la sostenibilidad de cafetales" (Altieri y Nicholls, 2002). Se evaluaron 8 cultivos considerados prevalentes en la zona: café, cacao, cítricos, plátano, coca, té, mango y papaya. Se consideró tres fincas para cada cultivo, con un total de 24 fincas evaluadas.

Con la participación de los productores, se seleccionaron diez indicadores prácticos de calidad de suelo y diez de salud del cultivo (Anexos 1 y 2), asignado un valor de 1 a 10 para cada indicador (siendo 1 el valor menos deseable, 5 un valor medio y $10 \mathrm{el}$ valor deseado), luego se obtuvieron los promedios. Los cultivos que obtuvieron valores menores a 5 se encuentran por debajo del umbral de sostenibilidad, no son sostenibles, por lo tanto requieren un manejo correctivo.
La metodología aquí presentada es una herramienta práctica para evaluar la sostenibilidad de cultivos y permite la participación directa del agricultor quien califica y asigna los valores numéricos a cada indicador.

La medición de la sostenibilidad de cultivos es una tarea necesaria, la selección acertada de indicadores es clave para buscar eficiencia en los resultados. Los valores de los indicadores son más fáciles de observar si se grafican en figuras tipo "ameba", esto permite visualizar de mejor manera el grado de sostenibilidad de los cultivos.

\section{Resultados y discusión}

\subsection{Tipología de productores}

Se ha determinado la existencia de tres tipos de productores en la región del Alto Urubamba: (En la Tabla 1 se puede ver sus principales características). 
Tipo I: Pequeños agricultores con cultivos perennes extensivos y de economía restringida. Comprende el $51.9 \%$ de la población (55 agricultores de la muestra); se dedican exclusivamente a la agricultura produciendo cultivos perennes (café, cacao, coca, té, frutales) para la venta y productos alimenticios (maíz, yuca, hortalizas) para el consumo, sus ingresos económicos son los más bajos de la zona.

Tipo II: Pequeños agricultores con actividad complementaria no agrícola y economía de subsidio.- Comprende el 24.5 $\%$ de la población (26 agricultores de la muestra); parte de su tiempo lo dedican a otras actividades no agrícolas (ganadero, comerciante, transportista, albañil, obrero municipal) para mejorar sus ingresos económicos.

Tipo III: Medianos agricultores con cultivos perennes intensivos y economía de capitalización.- comprende el $23.6 \%$ de la población (25 agricultores de la muestra); disponen de mayores recursos en la finca, tienen mayor área agrícola, más recursos financieros para la inversión, mejor nivel tecnológico y gestionan la finca con criterio empresarial.

\section{Tabla 1}

Características de los tipos de productores del Alto Urubamba, La Convención - Cusco.

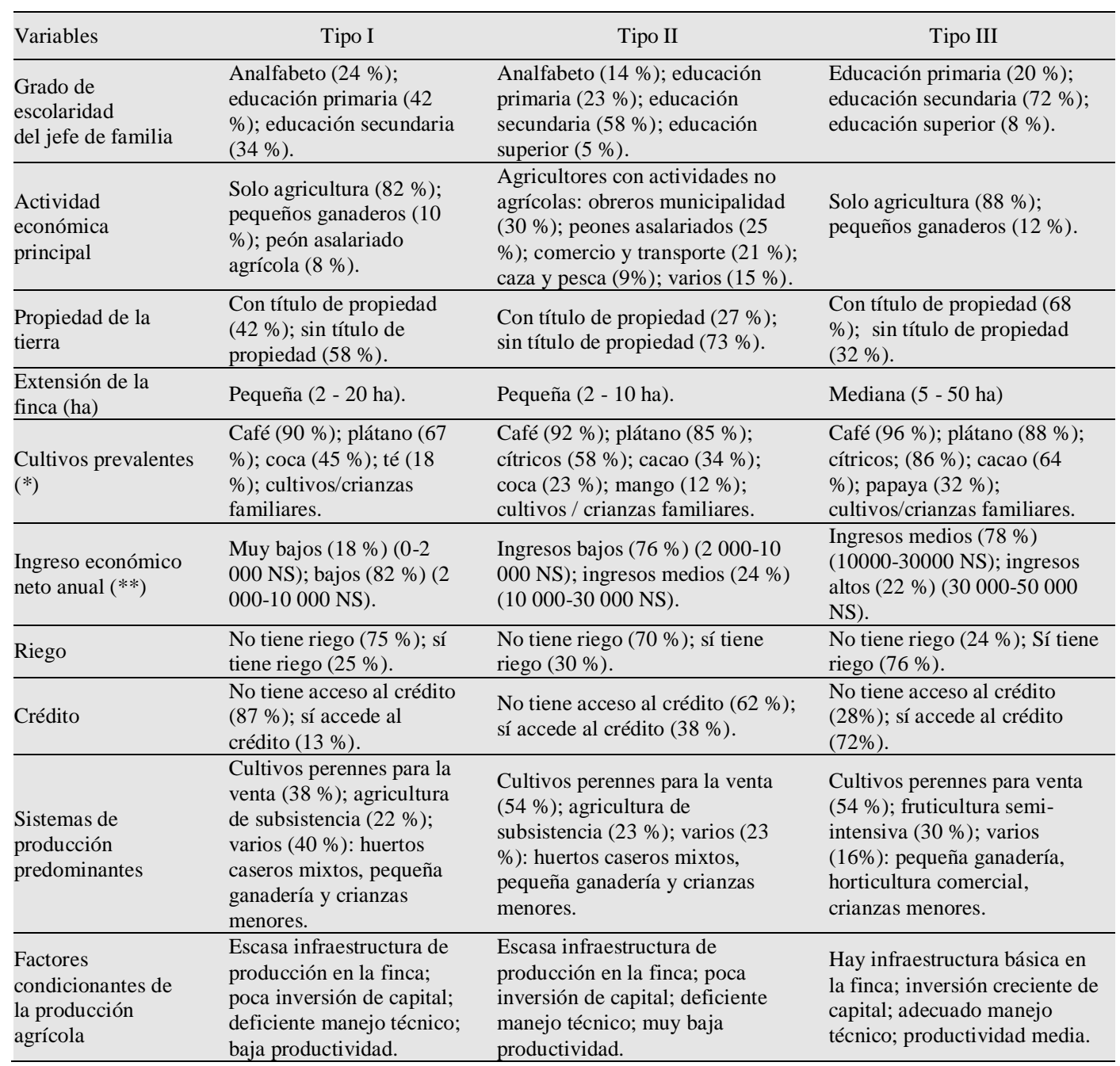

Fuente: Elaborado en base a encuesta para agricultores ( $n=106$, año 2008).

(*) Los porcentajes se refieren a la preferencia que tienen los agricultores por los cultivos.

(**) Tipo de cambio: \$ 1 Dólar = 2.90 Nuevos Soles (NS). 
En los tres tipos, la producción de los cultivos perennes se destinan principalmente para la venta $(85 \%)$; los cultivos transitorios se consumen más en la propia zona ( $80 \%$ de autoconsumo); la misma tendencia tienen las crianzas $(75 \%$ de autoconsumo).

Los recursos (tierra, mano de obra, bienes de capital, etc.) son utilizados por el agricultor en forma combinada para obtener una producción que satisfaga en primer lugar sus necesidades básicas y luego utiliza los excedentes para la venta (Tipo I y III). Cuando estos recursos están en mínima cantidad, la agricultura se vuelve una actividad poco o nada rentable; esta situación obliga a los miembros de la familia a mejorar sus ingresos con actividades fuera de la finca (Tipo II).

La familia siempre busca la reproducción de los recursos, de los que dispone (Apollin y Eberhart, 1999). En los tres tipos, la mayor o menor utilidad por la venta de los cultivos comerciales determina la situación económica de los agricultores $y$ en consecuencia su capacidad para comprar bienes y servicios que puedan reimpulsar los procesos agrícolas en la finca.

Las variables más importantes que determinaron la diferencia en los tipos encontrados fueron: la extensión de la finca, la actividad principal del productor, los cultivos prevalentes, el capital disponible y el nivel tecnológico del productor asociado con la gestión del predio.

La Figura 2 muestra la agrupación de las tres tipologías encontradas y el grado de afinidad entre los productores que conforman la muestra analizada.

Con el fin de realizar un análisis comparativo, se ha calculado el ingreso neto anual por familia (S/.) y la superficie de la finca (ha), con estos datos se ha graficado las tres tipologías encontradas, agrupando como "una nube de puntos" a los agricultores comprendidos en cada tipo (Figura 3).

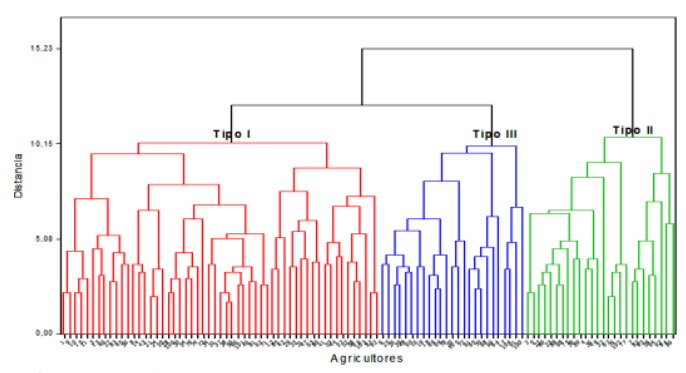

Figura 2. Agrupación de tres tipos de productores en el Alto Urubamba, La Convención - Cusco ( $\mathrm{n}=106$, año 2008).

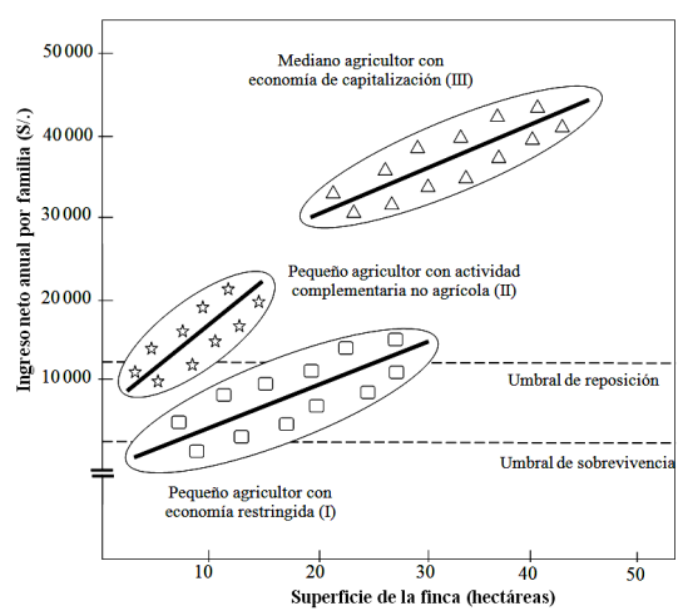

Figura 3. Representación gráfica de los tipos de productores en el Alto Urubamba, La Convención - Cusco ( $\mathrm{n}=106$, año 2008).

* Tipo de cambio: S/. 2.90 Nuevos Soles = \$ 1.00 Dólar Umbral de reposición $=$ S/.10950 (ingreso neto anual). Umbral de sobrevivência $=$ S/. 3650 (ingreso neto anual). $\mathrm{N}^{\circ}$ de miembros por familia $=4$ miembros (promedio) .

En la Figura 3 se observa la posición que toma cada tipo entre sí y respecto a los umbrales de "sobrevivencia" (nivel mínimo con el cual una familia puede sobrevivir) y "reposición" (mínimo necesario para satisfacer las necesidades de la familia y reponer los medios de producción) (Apollin y Eberhart, 1999). Para determinar el umbral de sobrevivencia se ha considerado el salario mínimo de un jornal (S/. 10.00) y para el umbral de reposición (S/. 30.00), valores vigentes en la zona para el año 2008. 
Los agricultores del tipo I se ubican entre ambos umbrales, lo que significa un estancamiento de los medios de producción y una condición económica precaria por la venta poco rentable de sus productos agropecuarios y la autosubsistencia. Los que se sitúan en el nivel inferior pueden llegar a abandonar la finca (migración) o vender su predio.

Los del tipo II apenas superan el umbral de reposición; los ingresos de fuera de la finca subsidian las necesidades básicas de la familia y les permiten hasta cierto punto, reponer sus medios de producción. En cambio la conducción de la finca queda desatendida y los niveles de producción disminuyen.

Los del tipo III superan ampliamente ambos umbrales debido al mayor ingreso económico familiar y a la explotación eficiente de sus agroecosistemas. Estos productores generan suficiente economía para reproducir con éxito los bienes de producción y de esa manera capitalizar su finca.

El análisis económico es necesario, porque como lo señala Yurjevic (1998) citado por Alvarado (2004) "la transición de la agricultura campesina hacia la sustentabilidad depende directamente de las inversiones que se hagan para mejorar la gestión social, productiva y económica, así como en los stocks de capital humano, natural productivo, social y construido".

En la zona es necesario convertir la agricultura tradicional de subsistencia en una agricultura comercial más competitiva, impulsada por pequeños empresarios integrados en cadenas de valor que vinculen a los productores con mercados más promisorios.

El Banco Mundial (2008) recomienda que para reducir la pobreza en los países en desarrollo, es necesario mejorar la disponibilidad de activos de los productores de las zonas rurales, hacer más competitiva y sostenible la agricultura en las fincas, diversificar las fuentes de ingreso orientándolas al mercado laboral.

\subsection{Evaluación de la sostenibilidad de cultivos}

Respecto a la calidad del suelo, la Tabla 2 muestra que los cultivos de cacao, café, té, plátano, mango y cítricos obtienen promedios superiores a 5, lo que otorga mayor sostenibilidad a la relación suelo planta; mientras que los cultivos de coca y papaya obtienen valores menores a 5 , lo que demuestra que ocupan suelos con limitaciones edáficas que los vuelve poco sostenibles.

En cuanto a la salud del cultivo, el mango, té y coca muestran mejores índices de sanidad vegetal, seguidos por el café y los cítricos; en cambio el cacao, plátano y papaya tienen promedios menores a 5 lo que demuestra que tienen limitaciones de sanidad en la zona.

Promediando ambos índices de calidad del suelo y salud del cultivo, se tiene que las fincas con té (6.65) y mango (6.50) obtienen promedios más altos de sostenibilidad, seguido de café y cacao (ambos con 6.25), cítricos (5.50), plátano (5.45) y coca (5.10). La papaya (4.60) muestra un promedio menor a cinco lo que califica a este cultivo como no sustentable para las condiciones en estudio.

Los valores de sostenibilidad en los cultivos evaluados se pueden considerar como "moderados o intermedios" si comparamos con otros estudios similares. En Turrialba Costa Rica, Altieri y Nicholls (2002) obtuvieron promedios combinados de 4.2 en cafetales convencionales en transición a orgánicos hasta 8.5 en cafetales orgánicos.

El desarrollo y uso de indicadores, resulta una herramienta adecuada y flexible para evaluar tendencias, establecer diferencias entre fincas y detectar los puntos críticos de manejo de recursos para el logro de una agricultura sustentable (Sarandón et al., 2004). 


\section{Tabla 2}

Evaluación de la sostenibilidad de cultivos prevalentes utilizando criterios de calidad del suelo y salud del cultivo en fincas del Alto Urubamba, La Convención - Cusco.

\begin{tabular}{lcccccccc}
\hline & Café & Cacao & Cítricos & Plátano & Coca & Té & Mango & Papaya \\
\cline { 2 - 8 } CALIDAD DEL SUELO & 7 & 7 & 5 & 7 & 4 & 5 & 4 & 4 \\
1. Estructura y textura & 8 & 7 & 7 & 6 & 7 & 8 & 7 & 8 \\
2. Compactación e infiltración & 6 & 8 & 6 & 6 & 5 & 6 & 8 & 3 \\
3. Profundidad del suelo & 6 & 8 & 4 & 6 & 4 & 6 & 5 & 4 \\
4. Estado de residuos & 8 & 8 & 5 & 7 & 4 & 7 & 4 & 4 \\
5. Materia orgánica, color y olor & 5 & 9 & 4 & 6 & 3 & 7 & 4 & 4 \\
6. Retención de humedad & 8 & 7 & 8 & 6 & 6 & 6 & 9 & 5 \\
7. Desarrollo de raíces & 9 & 7 & 5 & 5 & 3 & 9 & 4 & 4 \\
8. Cobertura del suelo & 8 & 9 & 8 & 6 & 2 & 7 & 7 & 4 \\
9. Erosión & 4 & 6 & 4 & 7 & 3 & 6 & 7 & 5 \\
10. Actividad biológica & 6.9 & 7.6 & 5.6 & 6.2 & 4.1 & 6.7 & 5.9 & 4.6 \\
\hline Promedio & & & & & & & & \\
\hline SALUD DEL CULTIVO & 7 & 5 & 6 & 5 & 6 & 6 & 9 & 5 \\
1. Apariencia & 6 & 5 & 6 & 5 & 7 & 6 & 8 & 5 \\
2. Crecimiento del cultivo & 4 & 4 & 5 & 3 & 9 & 8 & 9 & 3 \\
3. Tolerancia al estrés & 4 & 3 & 5 & 3 & 8 & 8 & 8 & 3 \\
4. Incidencia de enfermedades & 6 & 6 & 5 & 5 & 7 & 5 & 6 & 4 \\
5. Competencia por malezas & 6 & 4 & 7 & 4 & 6 & 8 & 7 & 6 \\
6. Rendimiento actual/potencial & 3 & 7 & 7 & 6 & 6 & 8 & 7 & 4 \\
7. Diversidad genética & 6 & 5 & 4 & 6 & 2 & 7 & 6 & 6 \\
8. Diversidad vegetal & 7 & 6 & 4 & 6 & 3 & 5 & 6 & 6 \\
9. Diversidad natural circundante & 7 & 4 & 5 & 4 & 7 & 5 & 5 & 4 \\
10. Sistema de manejo & 5.6 & 4.9 & 5.4 & 4.7 & 6.1 & 6.6 & 7.1 & 4.6 \\
\hline Promedio & & & & & & &
\end{tabular}

Fuente: Evaluaciones de campo.

Nota: Valores promedio menores a 5 indican sistemas no sostenibles.

Quizás lo más importante es que una vez aplicados los indicadores, cada productor puede visualizar el estado de su finca, observando qué atributos del suelo o de la planta andan bien o mal en relación al umbral preestablecido (Altieri y Nicholls, 2007). Esto es útil para que los agricultores entiendan porqué ciertas fincas se comportan mejor que otras, y qué hacer para mejorar los valores encontrados.

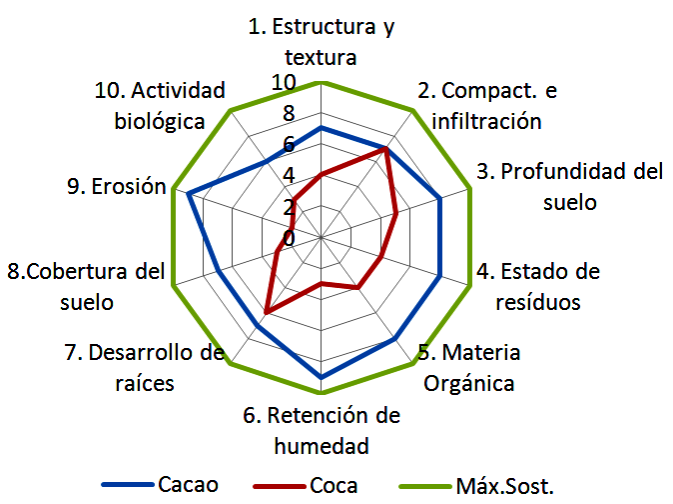

Figura 4. Comparación de indicadores de calidad de suelo en cacao y coca.

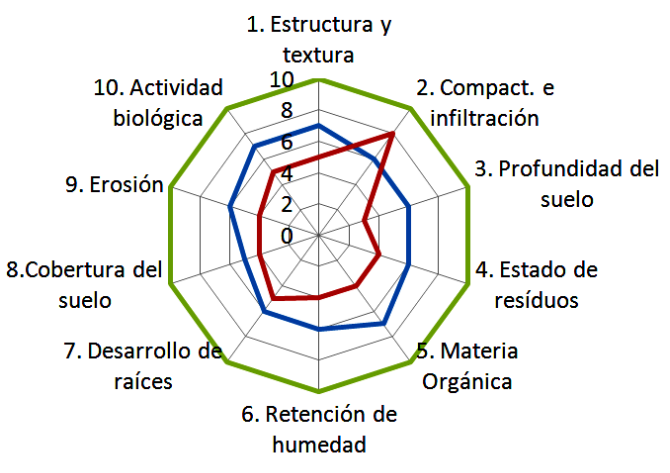

Figura 5. Comparación de indicadores de calidad de suelo en plátano y papaya.

Con los valores obtenidos para cada indicador se construyeron diagramas tipo "ameba", que permiten visualizar el estado general de la calidad del suelo y la salud del cultivo, tomando en cuenta que mientras más se aproxime la "ameba" al diámetro del círculo (valor 10: óptimo) el sistema es más sostenible (Figuras 4, 5, 6 y 7). Los cultivos de cacao, café y té muestran mejores índices de sustentabili- 
dad del suelo que los frutales y la coca, porque los primeros son cultivos pioneros establecidos en los mejores suelos de la región, de hábito perenne y con un manejo tradicional/orgánico que favorece la conservación del suelo. La coca es un cultivo tradicional de la zona, que tiene baja calificación debido a que está establecido en suelos marginales de laderas con baja calidad agrológica.

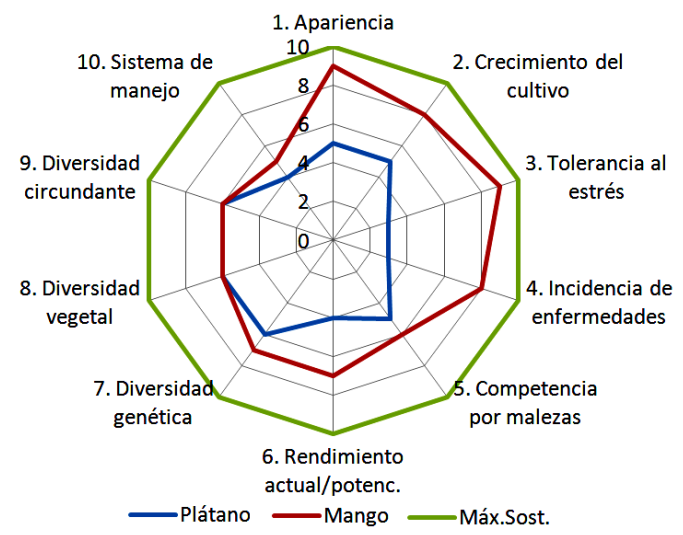
Figura 6. Comparación de indicadores de salud delcoca y papaya ocupan suelos con cultivo en plátano y mango.

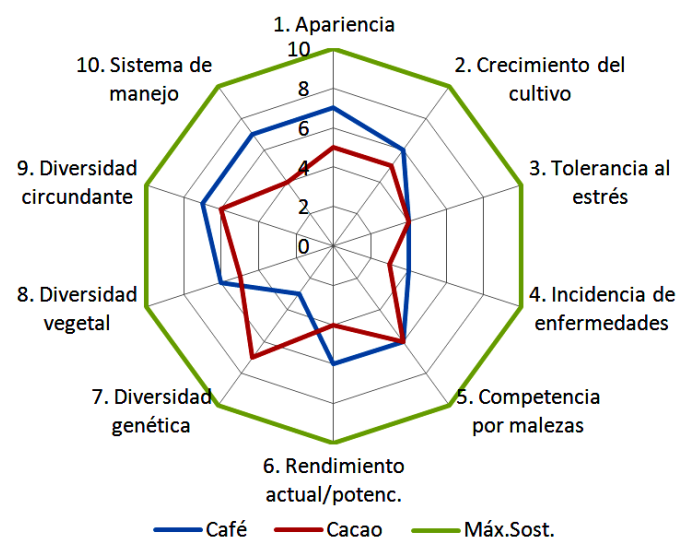

Figura 7. Comparación de indicadores de salud del cultivo en café y cacao.

\section{Conclusiones}

En el Alto Urubamba, La ConvenciónCusco se ha encontrado tres tipos de productores: tipo I (pequeños agricultores con cultivos perennes extensivos y de economía restringida); tipo II (pequeños agricultores con actividad complementaria no agrícola y economía de subsidio) y tipo
III (medianos agricultores con cultivos perennes intensivos y economía de capitalización). Las características descritas para cada tipo confirman la coexistencia de dos lógicas de producción: fincas de racionalidad capitalista y fincas de racionalidad tradicional o de subsistencia. Los productores del Tipo I se ubican entre los índices económicos de sobrevivencia y reposición, mantienen su condición de subsistencia debido al estancamiento de los medios de producción; los del Tipo II apenas superan el umbral de reposición, los ingresos de fuera de la finca subsidian las necesidades básicas de su familia y los del Tipo III generan suficiente economía para reproducir con éxito los bienes de producción y capitalizar su finca. La evaluación de sostenibilidad para calidad del suelo muestra que los cultivos de cacao, café, té, plátano, mango y cítricos son sostenibles (valor > 5), mientras que los cultivos de limitaciones edáficas (valor $<5$ ). Respecto a la salud del cultivo: el mango, té y coca muestran mejores índices seguidos por café y cítricos (todos > 5); en cambio el cacao, plátano y papaya (valor $<5$ ) presentan problemas fitosanitarios, lo que debe ser informado a las instituciones pertinentes a fin de que implementen programas integrados de control de plagas. Promediando ambos índices, se tiene que los cultivos de té (6.65) y mango (6.50) obtienen los valores más altos, luego café y cacao (6.25), cítricos (5.50), plátano (5.45) y coca (5.10). La papaya (4.60) se muestra como un cultivo no sustentable para las condiciones locales. Los indicadores utilizados en este estudio son fáciles $\mathrm{y}$ prácticos de utilizar por los productores, por lo que se recomienda aplicar esta metodología adaptando los procedimientos a las condiciones del lugar.

\section{Agradecimientos}

A los estudiantes de la Facultad de Ciencias Agrarias Tropicales de Quillabamba de la Universidad Nacional San Antonio Abad del Cusco, por su apoyo en la evaluación y a los productores de La Convención por la información de campo. 


\section{Referencias bibliográficas}

Altieri, MA.; Nicholls, CI. 2002. Un método agroecológico rápido para la evaluación de la sostenibilidad de cafetales. Manejo Integrado de Plagas y Agroecología 64: $17-24$

Altieri, M.A.; Nicholls, C.I. 2007. Conversión agroecológica de sistemas convencionales de producción: teoría, estrategias y evaluación. Rev. Ecosistemas. AEET. Universidad de California, Berkeley 16 (1).

Alvarado, de la F.F. 2004. Balance de la agricultura ecológica en el Perú 1980 - 2003. En: El problema Agrario en Debate - Sepia X Mesa Especial. Lima. Edit. SEPIA. 83 p.

Amador, M.; Durán, C.; Ruiz, VH.; Barquero, LC. 1995. Caracterización socio-productiva y tipología de productores del cantón de Acosta. Avances de Investigación $\mathrm{N}^{\circ}$ 14. Programa de Ecodesarrollo CEDECO. San José-Costa Rica. 34 p.

Apollin, F.; Eberhart, C. 1999. Análisis y diagnóstico de los sistemas de producción en el medio rural. Guía metodológica. CAMAREN, Quito-Ecuador. 239 p.

Banco Mundial. 2008. Informe sobre el desarrollo mundial 2008: Agricultura para el desarrollo. Bogotá, Colombia. Banco Mundial en coedición con Mayol Ediciones. S.A.

Conway, G.R. 1994. Sustainability in agricultural development: Trade-offs between productivity, stability and equitability. Journal for Farming Systems and Research-Extensions 4(2): 1-14.

Dixon, J.A.; Fallon, L.A. 1989. The concept of sustainability: origins, extensions and usefulness for policy. Society and Natural Resources 2: 73-84.

FAO / USAC. 1995. Caracterización del sistema agrario de la zona de retornados "Nueva Esperanza", Nentón, Huehuetenango. Informe de Proyecto. Guatemala. 66 p.

Fernández, CE.; Muschler, R. 1999. Aspectos de sostenibilidad de los sistemas de cultivo de café en América Central. En: Desafíos de la Caficultura en Centro América. Bertrand. B. Ed. San José, Costa Rica. IICA-PROMECAFE-CIRAD.

Girardin, P.; Bockstaller, C.; Van Der Werf, H. 1999. Indicators: tools to evaluate the environmental impacts of farming systems. Journal of Sustainable Agriculture 13(4): 5-17.
Gliessman, SR. 1998. Agroecology: ecological processes in sustainable agriculture. Michigan, Ann Arbor Press.

Gómez, AA.; Sweete, DE.; Syers, JK.; Couglan, KJ. 1996. Measuring sustainability of agricultural systems at the farm level. In Methods for assessing soil quality. SSSA Pub. 49.

Guzmán, C.G.I.; Alonso, M.AM. 2007. La investigación participativa en agroecología: una herramienta para el desarrollo sustentable. Ecosistemas 16(1): 1-12.

Hart, R. 1990. Componentes, subsistemas y propiedades del sistema finca como base para un método de clasificación. En: Escobar G. y Berdegué J. (eds) Tipificación de sistemas de producción agrícola. RIMISP/GLA. Santiago de Chile. 283 p.

López-Ridaura, S.; Van Keulen, H.; Van Ittersum, M.K.; Leffelaar, P.A. 2005. Multi-scale methodological framework to derive criteria and indicators for sustainability evaluation of peasant natural resource management systems. Environment, Development and Sustainability 7(1): 51-69.

Lovell, C.; Mandondo, A.; Moriarty, P. 2002. The question of scale in integrated natural resource management. Conservation Ecology. 5(2): 25.

Malagón, R.; Prager, M. 2001. El enfoque de sistemas: Una opción para el análisis de las unidades de producción agrícola. Universidad Nacional de Colombia. Sede Palmira. $190 \mathrm{p}$.

Masera, O.; Astier, M.; López-Ridaura, S. 1999. Sustentabilidad y manejo de recursos naturales. El Marco de Evaluación MESMIS. GIRA.A.C. México. $109 \mathrm{p}$.

Pacini, C.; Wossink, A.; Giesen, G.; Vazzana, C.; Huine, R. 2003. Evaluation of sustainability of organic, integrated and conventional farming systems: a farm and field-scale analysis. Agriculture Ecosystems and Environment. 95: 273-288.

Pretty, J. 2008. Agricultural sustainability: concepts, principles and evidence. Philosophical Transactions of the Royal Society B. 363: 447-465.

Sarandón; S.J.; Zuluaga, M.S.; Cieza, R.; Gómez, C.; Janjetic, L.; Negrete, E. 2004. Evaluación de la sustentabilidad de sistemas agrícolas de fincas en Misiones, Argentina, mediante el uso de indicadores. En Agroecología 1 (Sarandón SJ,ed.) Ediciones Científicas Americanas. p. 19-28. 
Anexo 1. Indicadores de calidad del suelo (Altieri y Nicholls, 2002).

\section{Estructura y textura}

Suelo polvoso, sin gránulos visibles (1)

Suelo suelto con pocos gránulos que se rompen al aplicar presión suave (5)

Suelo friable y granular, los agregados aún humedecidos mantienen la forma a la presión suave (10)

2. Compactación e infiltración

Compacto, se anega (1)

Presencia de capa compacta delgada, el agua se infiltra lentamente (5)

Suelo no compacto, el agua se infiltra fácilmente (10)

3. Profundidad del suelo

Subsuelo casi expuesto (1)

Suelo superficial delgado, con menos de $10 \mathrm{~cm}$ (5)

Suelo superficial más profundo, con más de $10 \mathrm{~cm}$ (10)

\section{Estado de Residuos}

Presencia de residuos orgánicos que no se descomponen o la hacen muy lentamente (1)

Se mantienen residuos del año anterior, en proceso de descomposición (5)

Residuos en varios estados de descomposición, residuos viejos bien descompuestos (10)

5. Color, olor y materia orgánica

Suelo pálido, con mal olor o químico, y no se observa la presencia de materia orgánica o humus (1)

Suelo pardo claro o rojizo, con poco olor y con algún grado de materia orgánica o humus (5)

Suelo de negro a pardo oscuro, con olor a tierra fresca, presencia abundante de materia orgánica y humus (10)

6. Retención de humedad

Suelo se seca rápido (1)

Suelo permanece seco durante la época seca (5)

Suelo mantiene humedad durante la época seca (10)

7. Desarrollo de raíces

Raíces poco desarrolladas, enfermas y cortas (1)

Raíces con crecimiento limitado, se observan algunas raíces finas (5)

Raíces con buen crecimiento, saludables y profundas, con abundante presencia de raíces finas (10)

8. Cobertura de suelo

Suelo desnudo (1)

Menos del 50\% del suelo cubierto por residuos, hojarasca o cubierta viva (5)

Más del 50\% del suelo con cobertura viva o muerta (10)

9. Erosión

Erosión severa, se nota arrastre de suelo y presencia de cárcavas y canalillos (1)

Erosión evidente, pero poca (5)

No hay mayores señales de erosión (10)

10. Actividad biológica

Sin signos de actividad biológica, no se observan lombrices o invertebrados (insectos, arañas) (1) Se observan algunas lombrices y artrópodos (5) Mucha actividad biológica, abundantes lombrices y artrópodos (10)
Anexo 2. Indicadores de salud del cultivo (Altieri y Nicholls, 2002).

\section{Apariencia}

Cultivo clorótico o descolorido, con signos severos de deficiencia de nutrimentos (1)

Cultivo verde claro, con algunas decoloraciones (5)

Follaje verde intenso, sin signos de eficiencia (10)

2. Crecimiento del cultivo

Cultivo poco denso, de crecimiento pobre. Tallos y ramas cortas y quebradizas, muy poco crecimiento de nuevo follaje (1)

Cultivo más denso, pero no uniforme, con crecimiento nuevo, ramas y tallos aún delgados (5) Cultivo denso, uniforme, buen crecimiento, con ramas y tallos gruesos y firmes (10)

3. Resistencia o tolerancia a estrés (sequía, lluvias intensas, plagas, etc.)

Susceptibles, no se recuperan bien después de un estrés (1)

Sufren en época seca o muy lluviosa, se recuperan lentamente (5)

Soportan sequía y lluvias intensas, recuperación rápida (10)

4. Incidencia de enfermedades

Susceptible a enfermedades, más del 50\% de plantas con síntomas (1)

Entre 20-50\% de plantas, con síntomas de leves a severos (5)

Resistentes, menos del $20 \%$ de plantas con síntomas leves (10)

5. Competencia por malezas

Cultivos estresados dominados por malezas (1)

Presencia media de malezas, cultivo sufre

competencia (5)

Cultivo vigoroso, se sobrepone a malezas, o malezas chapeadas no causan problemas (10)

6. Rendimiento actual o potencial

Bajo con relación al promedio de la zona (1)

Medio, aceptable con relación al promedio de la zona (5)

Bueno o alto, con relación al promedio de la zona (10)

\section{Diversidad genética}

Pobre, domina una sola variedad (1)

Media, dos variedades (5)

Alta, más de dos variedades (10)

8. Diversidad vegetal

Monocultivo sin sombra (1)

Con solo una especie de sombra (5)

Con más de dos especies de sombra, e incluso otros cultivos o malezas dominantes (10)

9. Diversidad natural circundante

Rodeado por otros cultivos, campos baldíos o carretera (1)

Rodeado al menos en un lado por vegetación natural (5)

Rodeado al menos en un $50 \%$ de sus bordes por vegetación natural (10)

10. Sistema de manejo

Monocultivo convencional, manejado con agroquímicos (1)

En transición a orgánico, con sustitución de insumos (5)

Orgánico diversificado, con uso de insumos orgánicos o biológicos (10) 
